\title{
INHIBITION OF CANDIDA ALBICANS GROWTH ON SURFACES TREATED BY DIELECTRIC BARRIER DISCHARGE WITH VARIOUS BARRIERS
}

\author{
JaN SLÁmA*, VÍTĚZSLAV KŘÍHA \\ Czech Technical University in Prague, Faculty of Electrical Engineering, Department of Physics, \\ Technická 2, 166 27, Prague 6, Czech Republic \\ * corresponding author: slamajan@fel.cvut.cz
}

\begin{abstract}
Discharges generating low temperature plasma at atmospheric pressure have the potential to treat surfaces biologically contaminated by organic matter in a non-destructive manner. We have been studying ways of inhibiting the growth of microorganisms with the use of dielectric barrier discharge (DBD) plasma. The effect of the choice of a barrier material and its thickness on the germicide properties of the DBD is described. We used Saboraud agar inoculated by $10^{5} \mathrm{cfu} / \mathrm{cm}^{2}$ of Candida albicans yeast as the model contaminated surface. After cultivation, the proportion of the treated surface with no $C$. albicans colony was evaluated.
\end{abstract}

Keywords: low temperature plasma, dielectric barrier discharge, inhibition, Candida albicans, treatment, biological surface.

\section{INTRODUCTION}

Discharges generating low temperature plasma at atmospheric pressure (e.g., corona discharge, dielectric barrier discharge (DBD), rf plasma jets, plasma needle, microwave discharges, gliding discharge (gliding arc, GlidArc)) are promising for the nondestructive treatment of biologically contaminated organic matter surfaces [1]. A specific discharge type is selected on the basis of its neither positive nor negative properties for the relevant biological application.

Generally, low temperature plasmas are known to be sources of chemically active agents (electrons, ions, electric fields, radicals and photons [2]) exploitable in industrial technologies (e.g., [3]) or in biological applications. Promising biological plasma applications using low temperature plasmas include liquid treatment [4, surface sterilization [5], inhibition processes [6] and cancer cell inactivation [7].

This study deals with adapting the electro-physical properties of DBD applied for Saboraud agar wafer surface decontamination and Candida albicans yeast inhibition. We have varied the dielectric barrier properties, e.g., thickness, which is equal to its dielectric strength $E_{\max }(\mathrm{kV} / \mathrm{mm})$ and the material that is used, i.e., mainly its permittivity $\varepsilon(\mathrm{F} / \mathrm{m})$. These parameters provide the overall character of the external electrical circuit, which consists of the DBD electrode system and power supply. The capacity of the DBD electrode system plays an essential role in the final impedance $Z$ of the system, ned it therefore has an influence on the circuit/discharge current. We have verified the hypothesis that the choice of various discharge barriers can change the yeast growth inhibition effect.

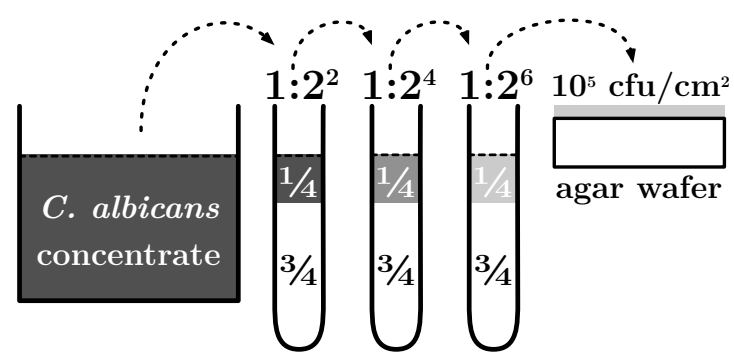

Figure 1. Dilution process for C. albicans fusion.

\section{Methods}

\subsection{Biologically CONTAMinated SuRfaCes}

As a model of a contaminated biological surface we prepared a set of Sabouraud agar substrates. They were realized as small wafers $22 \mathrm{~mm}$ in diameter and approximately $4 \mathrm{~mm}$ in thickness. The upper wafer surface was "contaminated" by a Candida albicans yeast solution.

The initial $C$. albicans suspension was diluted in three steps in a ratio of $1: 2^{2}$ (Fig. 1), so the final dilution ratio was $1: 2^{6}$. The $C$. albicans inoculum $50 \mu \mathrm{l}$ in volume was placed on each agar wafer. Each agar wafer was inoculated by approx. $10^{5} \mathrm{cfu} / \mathrm{cm}^{2}$ (colony forming units per square centimeter) of $C$. albicans yeast, which represented a model of biological contamination. The agar wafers were stored in sterile conditions in a fridge during the time before treatment to slow down the $C$. albicans growth (similar growth/activity mechanism are mentioned by Fang [8]). 


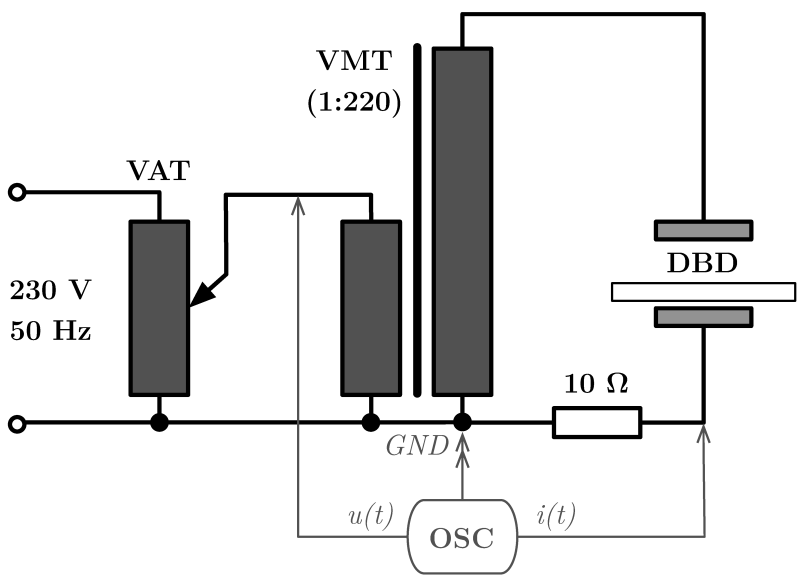

Figure 2. The electric circuit scheme with a capacitive load (the DBD reactor), the oscilloscope (OSC) and the $10 \Omega$ shunt resistor.

\subsection{Plasma treatment}

The apparatus (Fig. 2 consisted of the supplying part and the part with an electrode system. The electrodes ("HV" and "GND") of the DBD reactor (Fig. 3) were powered by the voltage measuring transformer (VMT) with input : output voltage ratio $\frac{22000 \mathrm{~V}}{\sqrt{2}}: \frac{100 \mathrm{~V}}{\sqrt{2}}$, i.e., transformation ratio $1: 220$ (for a detailed description, see [9]). The high voltage side of the VMT was connected to the DBD reactor electrodes, and the low voltage side was connected to the variable autotransformer (VAT) as a voltage regulator in the range $0 \div 250 \mathrm{~V}$. The autotransformer was powered by AC voltage $230 \mathrm{~V} / 50 \mathrm{~Hz}$. The electrical values (e.g., voltage $u(t)$ on the primary winding of the VTM and the discharge current $i(t)$ as a portrait of the voltage on a $10 \Omega$ shunt resistor) were measured using a $200 \mathrm{MHz}$ OWON PDS 8102T oscilloscope.

The electrode system (Fig. 3) consisted of a single circular plane electrode (GND) approx. $36 \mathrm{~mm}$ in diameter covered by a dielectric barrier of thickness $T$. The opposite electrode (HV) was placed at a distance $D$ above the grounded electrode. This electrode was realized as a set of 7 spikes placed into a symmetric triangular grid (isometric composition). The step of the grid was $5 \mathrm{~mm}$; the spike in the center was dropped by about $1 \mathrm{~mm}$.

Six different dielectric barriers were used, with a range of materials and dimensions (values of $\varepsilon_{\mathrm{r}}$ are taken from [10]):

- polyethylene terephthalate (PET) foil, $\varepsilon_{\mathrm{r}}=2.3,0.1 \times 105 \times 105 \mathrm{~mm}$;

- polystyrene (PS) Petri dish, $\varepsilon_{\mathrm{r}}=2.5,0.9 \times \varnothing 90 \mathrm{~mm}$;

- polymethyl methacrylate (PMMA), $\varepsilon_{\mathrm{r}}=3.0,2.1 \times 94 \times 144 \mathrm{~mm}$;

- glass, $\varepsilon_{\mathrm{r}}=7.5,1.1 \times 95 \times 89 \mathrm{~mm}$;

- glass, $\varepsilon_{\mathrm{r}}=7.5,2.8 \times 119 \times 119 \mathrm{~mm}$;

- glass, $\varepsilon_{\mathrm{r}}=7.5,5.0 \times 170 \times 148 \mathrm{~mm}$.
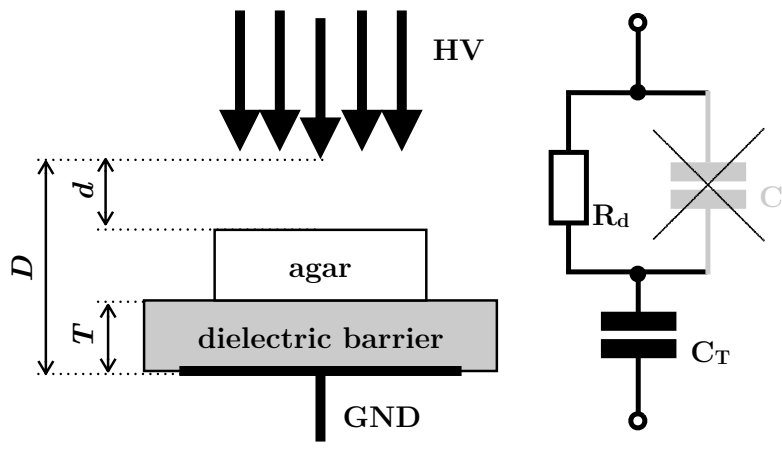

FiguRE 3 . The DBD reactor with a grounded electrode (GND) and a high voltage electrode (HV), a dielectric barrier and a slice of agar in between them on the left; the equivalent circuit on the right.

\begin{tabular}{clccr}
\hline Group & \multicolumn{1}{c}{ Barrier } & $\begin{array}{c}\varepsilon_{\mathrm{r}} \\
(-)\end{array}$ & $\begin{array}{c}\varepsilon_{\mathrm{r}} / T \\
\left(\mathrm{~mm}^{-1}\right)\end{array}$ & $I_{\mathrm{RMS}}$ \\
\hline C & $2.1 \mathrm{~mm}$ PMMA & 3.0 & 1.4 & $49 \mathrm{~mA}$ \\
& $5.0 \mathrm{~mm}$ glass & 7.5 & 1.5 & $55 \mathrm{~mA}$ \\
\hline \multirow{2}{*}{ B } & $2.8 \mathrm{~mm}$ glass & 7.5 & 2.7 & $44 \mathrm{~mA}$ \\
& $0.9 \mathrm{~mm}$ PS & 2.5 & 2.8 & $53 \mathrm{~mA}$ \\
\hline \multirow{2}{*}{ A } & $1.1 \mathrm{~mm}$ glass & 7.5 & 6.8 & $73 \mathrm{~mA}$ \\
& $0.1 \mathrm{~mm}$ PET & 2.3 & 23 & $143 \mathrm{~mA}$ \\
\hline
\end{tabular}

TABle 1. Relative permittivity, current and the $\varepsilon_{\mathrm{r}} / T$ ratio values for each barrier sorted into groups $\mathbf{A}, \mathbf{B}$ and $\mathbf{C}$.

An agar slice approximately $4 \mathrm{~mm}$ in thickness and $20 \mathrm{~mm}$ in diameter was placed on the dielectric barrier. During all expositions, a constant distance $d=11 \mathrm{~mm}$ was maintained between the spikes and the upper surface of the agar slice, which had already been inoculated with $C$. albicans yeast. The electrode system was powered by the AC voltage $U_{\mathrm{RMS}}=13.2 \mathrm{kV}(50 \mathrm{~Hz}$ sine wave $)$ for each exposition time $t_{\exp }=1,2,4,8,16,24 \mathrm{~min}$. The RMS values of the discharge current $I_{\mathrm{RMS}}$ were captured by the oscilloscope during expositions $t_{\text {exp }}$. The average values of $I_{\mathrm{RMS}}$ (calculated from multiple records corresponding to $\left.t_{\exp }\right)$ were logged into Tab. 1 .

\subsection{Cultivation, LOgGing And Storing}

After plasma surface treatment, the agar wafers were stacked on an empty Petri dish (Fig. 4) which was placed into the thermostatic chamber. There, the surviving $C$. albicans colony forming units were cultivated for 48 hours at temperature $37^{\circ} \mathrm{C}$. The final step was to evaluate the $C$. albicans inhibition.

\subsection{EQUiVALENT CIRCUIT}

The electrical circuit for DC or low frequency AC powering $(f=50 \mathrm{~Hz})$ with two serial capacitors $C_{\mathrm{d}}$ and $C_{\mathrm{T}}$ was used as a model of an air gap $d$ and a dielectric barrier of a thickness $T$ (Fig. 3). The resistivity of the agar structure could be neglected in 


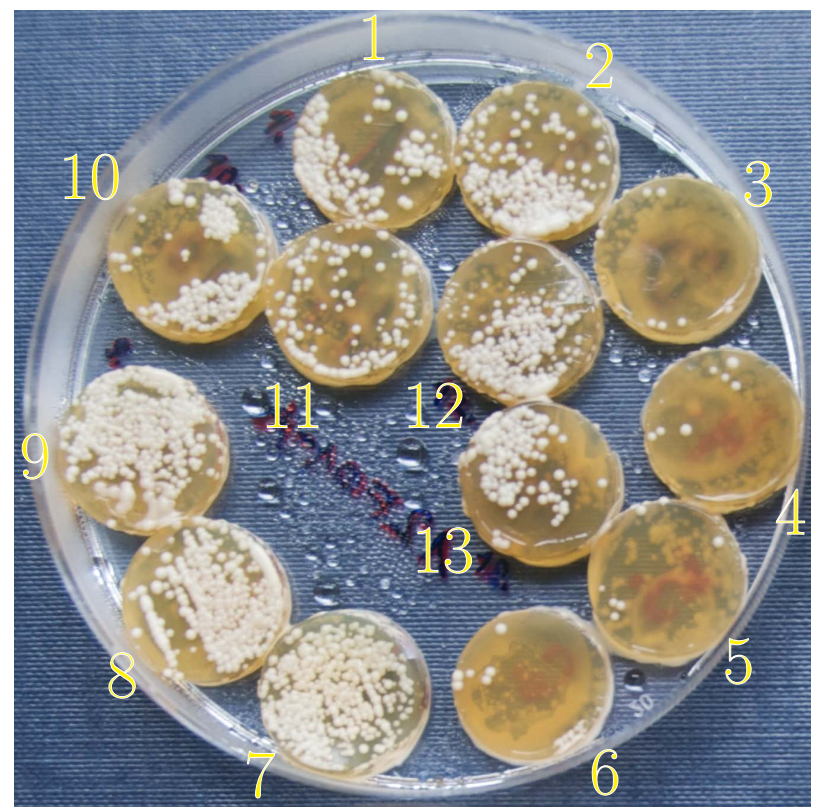

Figure 4. An example of the agar wafers on a Petri dish; the $0.1 \mathrm{~mm}$ PET foil (see group A in Figs. 5 and 6) and $5.0 \mathrm{~mm}$ glass (group $\mathbf{C}$ ) dielectric barrier treatment results; the white bullets are $C$. albicans colonies.

this simplified case. Firstly, the voltage estimation was based on the equation for the capacity of a planar capacitor

$$
C=\varepsilon_{0} \varepsilon_{\mathrm{r}} \frac{S}{d},
$$

where $\varepsilon_{0}(\mathrm{~F} / \mathrm{m})$ is the permittivity of the vacuum, $\varepsilon_{\mathrm{r}}$ (-) is the relative permittivity, $S\left(\mathrm{~m}^{2}\right)$ is the surface area of the electrode and $d(\mathrm{~m})$ is the inter electrode distance. Further, we used the relation $Q=C U$ (C; F, V) for a DC circuit; the relation for the capacitive impedance $\hat{X}_{\mathrm{C}}=(\mathbf{i} \omega C)^{-1}(\Omega)$ for an AC circuit, where $\omega=2 \pi f(\mathrm{rad} ; \mathrm{Hz})$ and $\mathbf{i}$ is the imaginary unit. More than $95 \%$ of the total voltage (supplied by the VTM, Fig. 2) was present on the air gap $d$ (valid for all six dielectrics, it is expected DC or low frequency $\mathrm{AC}$ powering). For further approximate consideration the air gap capacity $C_{\mathrm{d}}$ was therefore neglected. Only barrier capacity $C_{\mathrm{T}}$ (i.e., capacitance $\hat{X}_{C_{\mathrm{T}}} \sim 1 / \frac{\varepsilon_{\mathrm{r}}}{T}$ ratio) was considered in the following text. For our estimation we used only the module

$$
Z=\left(\left(R_{\mathrm{d}}\right)^{2}+\left(\hat{X}_{C_{\mathrm{T}}}\right)^{2}\right)^{1 / 2}
$$

of the DBD reactor complex impedance $\hat{Z}=R_{\mathrm{d}}+\hat{X}_{C_{\mathrm{T}}}$, where $R_{\mathrm{d}}$ is a non linear circuit element equal to the resistance of a DBD filament discharge channel serially connected to $\hat{X}_{C_{\mathrm{T}}}$.

During the evolution of the DBD the discharge region resistance $R_{\mathrm{d}}$ drops dramatically from a nonconductive insulator $\left(R_{\mathrm{d}} \rightarrow \infty\right)$ to an ionized conductive substance $\left(R_{\mathrm{d}} \rightarrow 0\right)$. This process is shown in Fig. 5. The left quarter is for low ionized states, where the resistance $R_{\mathrm{d}}$ has a significant influence

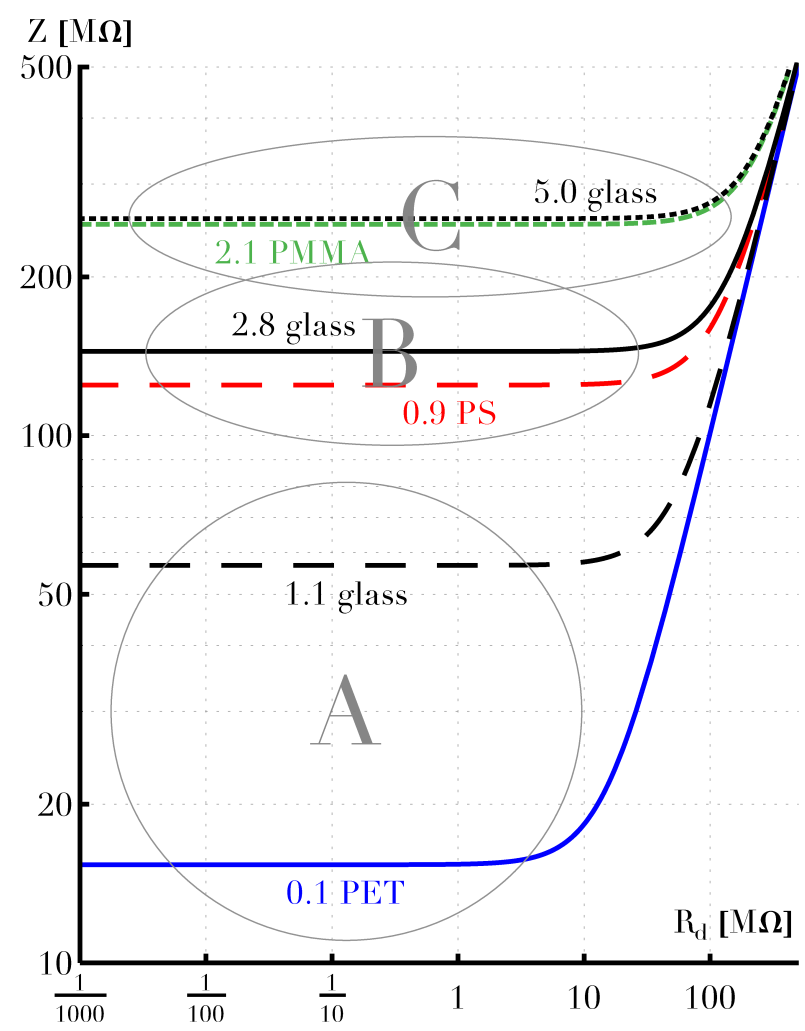

Figure 5. Estimated total impedance $Z$ dependence on the resistivity of discharge area $R_{\mathrm{d}}$.

on the overall impedance $Z$; by contrast, the right side of the chart is for the state where only capacitive impedance $\hat{X}_{C_{\mathrm{T}}}$ plays a dominant role. The values of impedance $Z$ are in good accordance with the "pointto-plane" electrode configuration DC corona discharge experiments [11].

\section{RESULtS}

An example of the treatment results for a PET foil barrier (specimens 1-6), for $5.0 \mathrm{~mm}$ glass (specimens 8-13) and control specimen 7 is shown in Fig. 4 Each set of six specimens is ordered sequentially from the shortest to the longest exposition time, i.e., $t_{\exp }=1,2,4,8,16,24 \mathrm{~min}$. The other samples are not shown for the reasons of space. The final treatment results are summarized in chart in Fig. 6. It shows the dependence of area covered by $C$. albicans yeast colonies (value "1", i.e., $100 \%$ corresponds to full coverage, i.e., no positive treatment effect was registered) on exposition time $t_{\text {exp }}$.

The experimental results can be divided into three groups, marked as $\mathbf{A}, \mathbf{B}$ and $\mathbf{C}$, characterized by the tendencies/slopes in Fig. 6. An analogous situation is shown in Fig. 5 and a certain parallelism can be observed in the $I_{\text {RMS }}$ values in Tab. 1 . Similarly the $\varepsilon_{\mathrm{r}} / T$ ratio (which is in substance the partially expressed capacity of the barrier normalized to a unit surface) also corresponds with observed trends.

The estimated treatment time for reaching $\mathrm{LD}_{50}$ (median lethal dose) was less than 2 minutes for the 

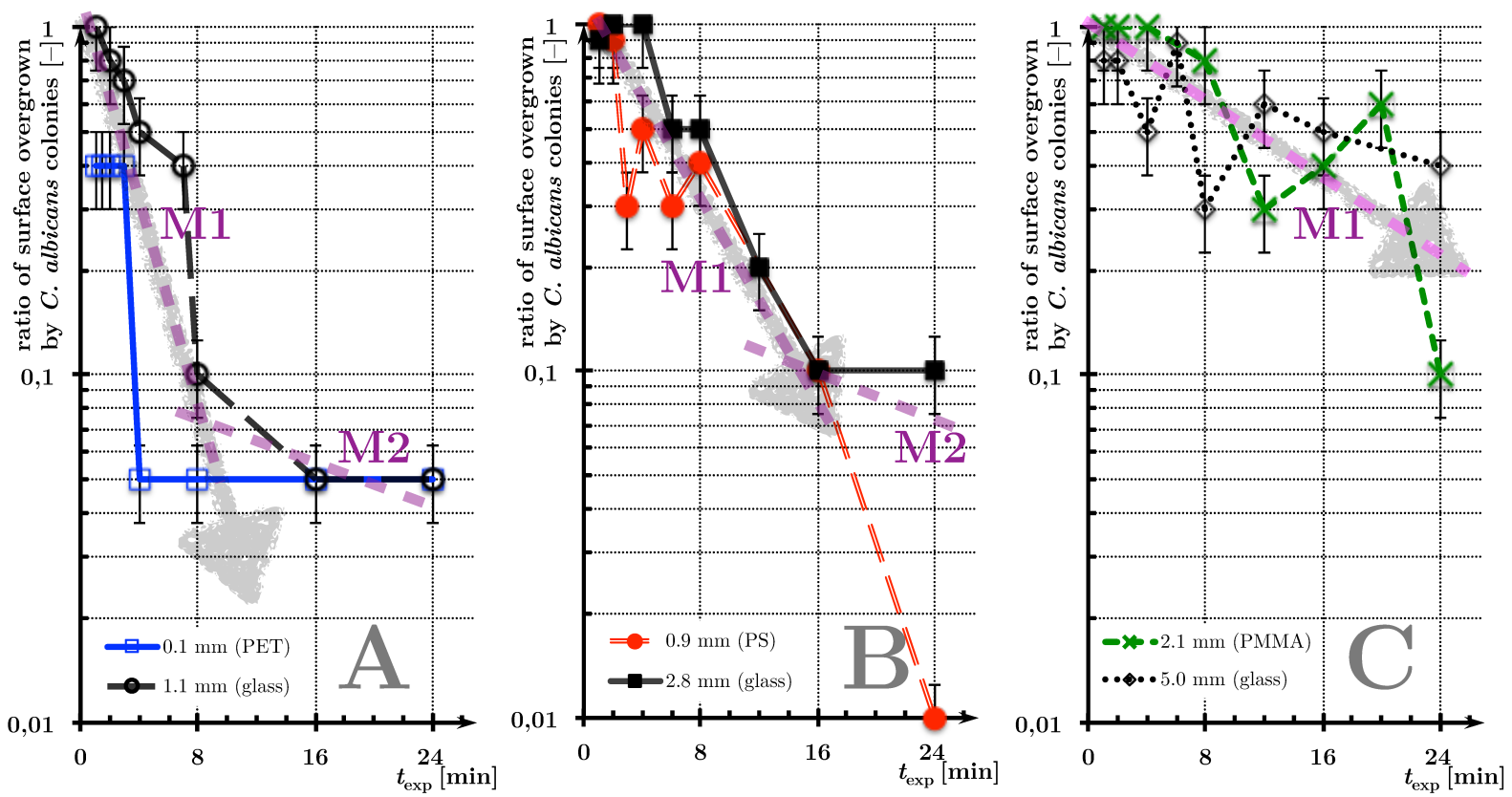

FiguRE 6. Dependence of C. albicans growth on time exposition of the agar surface; trends are expressed by gray arrows, and the expected inhibition phases are marked by magenta dashed lines (marked as line M1 and line M2, respectively).

$0.1 \mathrm{~mm}$ PET barrier, 6 minutes for the $1.1 \mathrm{~mm}$ glass barrier, 8 minutes for the $0.9 \mathrm{~mm}$ PS and $2.8 \mathrm{~mm}$ for the glass barriers. The results using the barriers in group $\mathbf{C}$ lay around the $\mathrm{LD}_{50}$ value in time $t_{\exp } \in$ $\langle 10,20\rangle$ min. There were high values of impedance $Z$ (group C) when the discharge was switching between several burning modes treating the agar surface nonhomogeneously. This effect may explain the oscillating character of results in group $\mathbf{C}$.

Parallel to the results mentioned above, one can find similarities to Moisan's explanations of plasma sterilization mechanisms [12. Moisan describes the mechanism of plasma inhibition by two processes: UV inactivation and erosion. UV inactivation plays an important role in the first phase of the inhibition process (see M1 in Fig. 6), while erosion acts significantly in the second phase (M2). Both, the first and the second inhibition process phase can probably be found in groups $\mathbf{A}$ and $\mathbf{B}$. In group $\mathbf{C}$, there is no significant trend similar to the second inhibition phase. We did not observe the third phase of plasma inhibition (i.e., UV inactivation) described by Moisan 12 for longer exposition times $\left(t_{\exp }>24 \mathrm{~min}\right)$.

\section{Conclusion}

The results of the study show that the choice of barrier thickness $T$ and the materials of the barrier can be used for adjusting the duration of the inactivation treatment. This effect cannot be explained by a simple change of the discharge current. In the case of groups $\mathbf{B}$ and $\mathbf{C}$, the discharge currents are comparable although the inactivation process for group $\mathbf{B}$ seems to be faster than for group C. The outstanding treatment results in group $\mathbf{A}$ seemed to be due to the synergy of the high current $I_{\mathrm{RMS}}$ values and small thicknesses $T$. The separate role of the barrier thickness, the barrier construction and the discharge currents on the inhibition effect, and their synergies, could be a subject for further investigation.

Analogous trends were found between measured growth dependence (Fig. 6) and Moisan's explanation of plasma sterilization mechanisms, i.e., the survival curve which is characteristic for plasma sterilization [12].

\section{ACKNOWLEDGEMENTS}

This work was supported by CTU in Prague grant SGS13/194/OHK3/3T/13.

\section{REFERENCES}

[1] Šerá B. et al: How various plasma sources may affect seed germination and growth. $13^{\text {th }}$ International Conference on Optimization of Electrical and Electronic Equipment (OPTIM), 2012, p. 1365-1370.

[2] Sakiyama Y, Graves D.: Atmospheric pressure gas plasmas for biomedical applications. Online:

http://www.graveslab.org/lab-resources/ presentations/20110512_NCCAVS_YSakiyama.pdf [2014-08-12].

[3] Klenko Y., Pichal J.: $\mathrm{TiO}_{x}$ thin films AP DBD deposition on polyamide rope. Problems of Atomic Science and Technology, 6, 2010, p. 191-193.

[4] Scholtz V. et al: Comparison of point-to-plane and point-to-point corona discharge for the decontamination or sterilization of surfaces and liquids. Plasma Medicine, 1 (1), 2011, p. 21-25. DOI:10.1615/PlasmaMed.v1.i1.20

[5] Taran V.S. et al.: Investigation of Pulsed Barrier Discharge in Water-Air Gap. Problems of Atomic Science and Technology, 83 (19), 2013, p. 249-251. 
[6] Bujaček K. et al.: Decontamination Effects of the Corona Discharge with Plane to Bent Needle

Configuration. Problems of Atomic Science and Technology, 82 (18), 2012, p. 187-189.

[7] Vandamme M.: Response of Human Glioma U87 Xenografted on Mice to Non Thermal Plasma Treatment. Plasma Medicine, 1 (1), 2011, p. 7-43.

[8] S. H. Fang et al: Evidence for arylamine $N$-acetyltransferase activity in the fungi Candida albicans. Toxicology Letters, 92 (2), 1997, p. 109-116. DOI:10.1016/S0378-4274(97)00037-4

[9] Sláma J.: Napájeni atmosférického dielektrického bariérového výboje. Praha, 2007.
[10] Dielectric Constants Chart. Online: http://www.asiinstr.com/technical/Dielectric\% 20Constants.htm [2012-05-01]. Available at: http://web.archive.org/web/20120703035752/http: /www.asiinstr.com/technical/Dielectric\% 20Constants.htm [2014-08-12].

[11] Horák J., Khun J.: Impedance-stabilized positive corona discharge and its decontamination properties. Journal of Physics: Conference Series, 223, 2010, 012006. DOI:10.1088/1742-6596/223/1/012006

[12] Moisan M. et al.: Plasma sterilization. Methods and mechanisms. Pure Appl. Chem., 74 (3), 2002, p. 349-358. DOI:10.1351/pac200274030349 